\title{
Deciphering the peculiar behavior of beta-lapachone in lipid monolayers and bilayers
}

Xiao Wu${ }^{1}$, Benjamin Chantemargue ${ }^{2,3}$, Florent Di $\mathrm{Meo}^{2}$, Claudie Bourgaux ${ }^{1}$, David Chapron ${ }^{1}$, Patrick Trouillas $^{2,3}$, Veronique Rosilio $^{1 *}$

${ }^{1}$ Institut Galien Paris Sud, Univ. Paris-Sud, CNRS, Université Paris-Saclay, 92296, ChâtenayMalabry, France.

${ }^{2}$ INSERM U1248, Faculty of Pharmacy, Université de Limoges, 2 rue du Docteur Marcland, 87025 Limoges Cedex, France.

${ }^{3}$ Centre of Advanced Technologies and Materials, Department of Physical Chemistry, Faculty of Science, Palacký University, tr. 17 listopadu 12, 77146 Olomouc, Czech Republic.

* To whom correspondence should be addressed. Email: veronique.rosilio@u-psud.fr 


\section{Force field parameters of b-lap (prepi and frcmod files)}

\subsection{Prepi file}

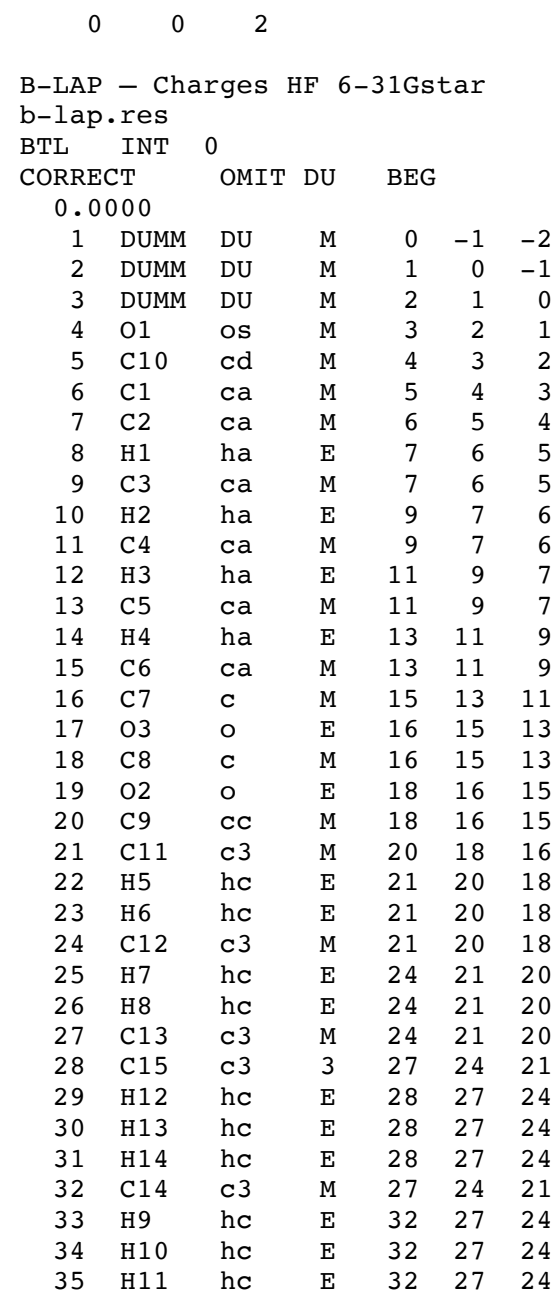

$\begin{array}{lrrr}0.000 & .0 & .0 & .00000 \\ 1.449 & .0 & .0 & .00000 \\ 1.523 & 111.21 & .0 & .00000 \\ 1.540 & 111.208 & -180.000 & -0.212300 \\ 1.349 & 42.020 & -177.524 & 0.021900 \\ 1.476 & 112.713 & 2.409 & -0.029300 \\ 1.400 & 121.184 & -0.593 & -0.074300 \\ 1.083 & 119.371 & 0.129 & 0.115800 \\ 1.397 & 120.234 & -179.898 & -0.141500 \\ 1.087 & 119.447 & 179.872 & 0.157000 \\ 1.396 & 120.519 & -0.232 & -0.161900 \\ 1.086 & 120.230 & -179.927 & 0.159800 \\ 1.394 & 119.640 & 0.052 & -0.119100 \\ 1.085 & 121.593 & -179.771 & 0.163200 \\ 1.398 & 120.313 & 0.157 & -0.054500 \\ 1.487 & 119.496 & -179.856 & 0.489900 \\ 1.218 & 122.630 & 0.547 & -0.473500 \\ 1.550 & 117.577 & -179.140 & 0.385400 \\ 1.224 & 118.979 & 178.704 & -0.483500 \\ 1.459 & 118.365 & -0.904 & -0.035500 \\ 1.506 & 118.167 & 178.283 & -0.118200 \\ 1.098 & 110.282 & 74.928 & 0.071800 \\ 1.095 & 108.984 & -40.147 & 0.071800 \\ 1.531 & 110.282 & -162.009 & -0.114800 \\ 1.097 & 109.568 & 74.650 & 0.057400 \\ 1.096 & 110.971 & -167.348 & 0.057400 \\ 1.536 & 111.854 & -45.329 & 0.345500 \\ 1.533 & 112.850 & -63.281 & -0.173500 \\ 1.094 & 111.611 & 58.889 & 0.051600 \\ 1.096 & 110.182 & -61.292 & 0.051600 \\ 1.094 & 110.269 & 179.096 & 0.051600 \\ 1.528 & 111.576 & 170.667 & -0.316000 \\ 1.094 & 110.585 & -176.093 & 0.085400 \\ 1.095 & 110.116 & 64.116 & 0.085400 \\ 1.095 & 110.648 & -55.874 & 0.085400\end{array}$

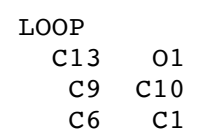

$\begin{array}{llrr}\text { IMPROPER } & & \\ \text { C1 } & \text { C9 } & \text { C10 } & \text { O1 } \\ \text { C6 } & \text { C2 } & \text { C1 } & \text { C10 } \\ \text { C1 } & \text { C3 } & \text { C2 } & \mathrm{H} 1 \\ \text { C4 } & \text { C2 } & \text { C3 } & \text { H2 } \\ \text { C5 } & \text { C3 } & \text { C4 } & \text { H3 } \\ \text { C4 } & \text { C6 } & \text { C5 } & \text { H4 } \\ \text { C7 } & \text { C5 } & \text { C6 } & \text { C1 } \\ \text { C8 } & \text { C6 } & \text { C7 } & \text { O3 } \\ \text { C7 } & \text { C9 } & \text { C8 } & \text { O2 } \\ \text { C8 } & \text { C11 } & \text { C9 } & \text { C10 }\end{array}$

DONE

STOP 


\subsection{Fremod file}

remark goes here MASS

BOND

ANGLE

$$
\text { DIHE }
$$

$\begin{array}{lllll}\text { ca-cd-os-c3 } & 1 & 1.050 & 180.000 & 2.000 \\ \text { ca-ca-cd-cc } & 1 & 0.700 & 180.000 & 2.000 \\ \text { ca-ca-cd-os } & 1 & 0.700 & 180.000 & 2.000 \\ \text { cc-cd-os-c3 } & 1 & 1.050 & 180.000 & 2.000\end{array}$

IMPROPER

ca-ca-ca-cd
ca-ca-ca-ha

1.1

180.0

$1.1 \quad 180.0$

2.0

c -ca-ca-ca

180.0
180.0

2.0

10.5

180.0
180.0

2.0

$\begin{array}{lll}\mathrm{C} & -\mathrm{Ca}-\mathrm{C}-\mathrm{O} \\ \mathrm{C}-\mathrm{C}-\mathrm{C}-\mathrm{O}\end{array}$

c $-\mathrm{c} 3-\mathrm{Cc}-\mathrm{cd}$

10.5

180.0

2.0

2.0

2.0

ca-cc-cd-os

180.0

2.0

NONBON 


\section{Raw results from molecular dynamics simulations}
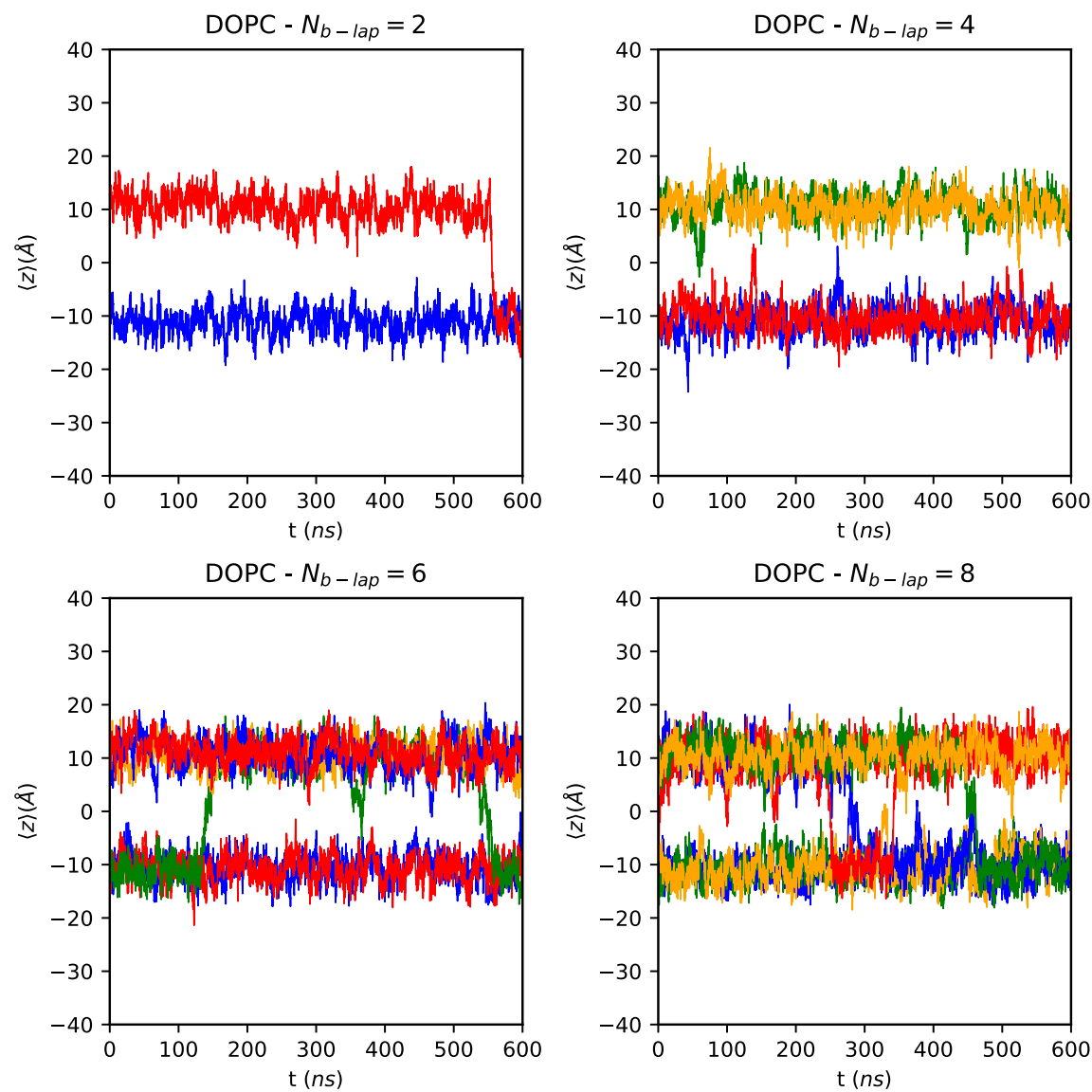

Figure Sth1. Evolution of the distance between b-lap and lipid bilayer centers of mass over the 600 ns $(\mathrm{N}, \mathrm{P}, \mathrm{T}) \mathrm{MD}$ simulations in pure DOPC lipid bilayer membranes. 

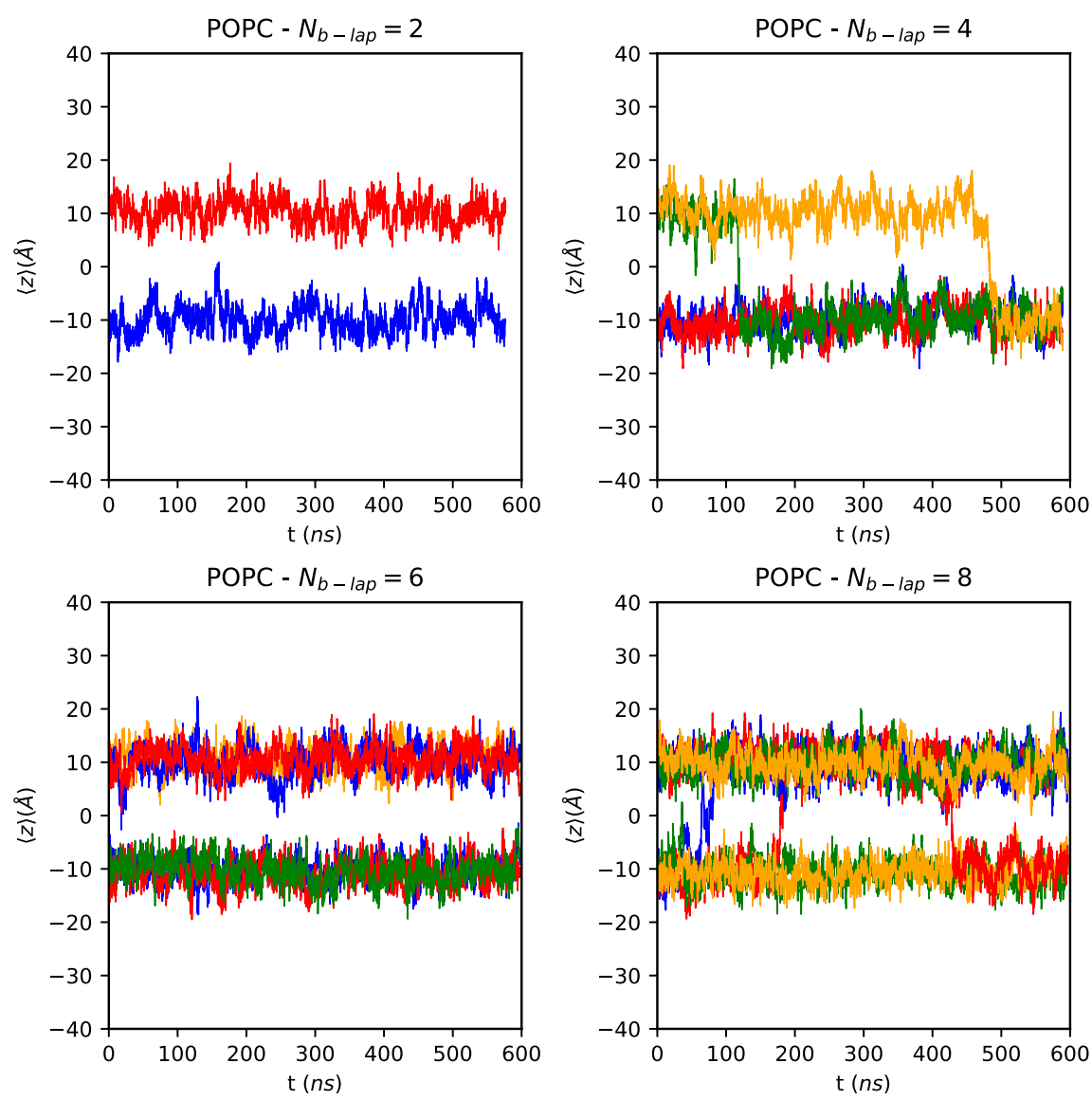

Figure Sth2. Evolution of the distance between b-lap and lipid bilayer centers of mass over the $600 \mathrm{~ns}(\mathrm{~N}, \mathrm{P}, \mathrm{T}) \mathrm{MD}$ simulations in pure POPC lipid bilayer membranes. 

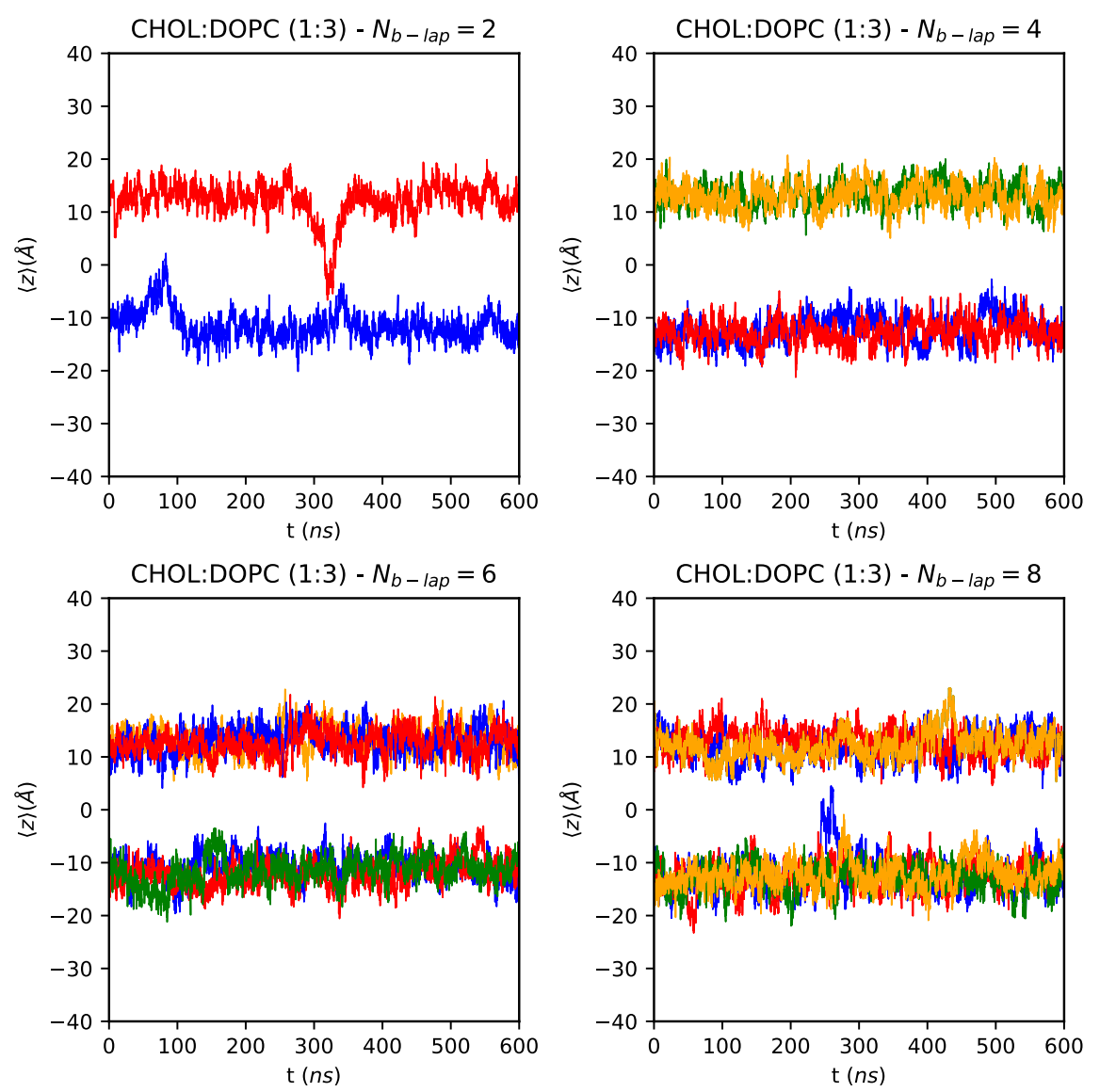

Figure Sth3. Evolution of the distance between b-lap and lipid bilayer centers of mass over the $600 \mathrm{~ns}(\mathrm{~N}, \mathrm{P}, \mathrm{T}) \mathrm{MD}$ simulations in pure CHOL:DOPC (1:3) lipid bilayer membranes. 

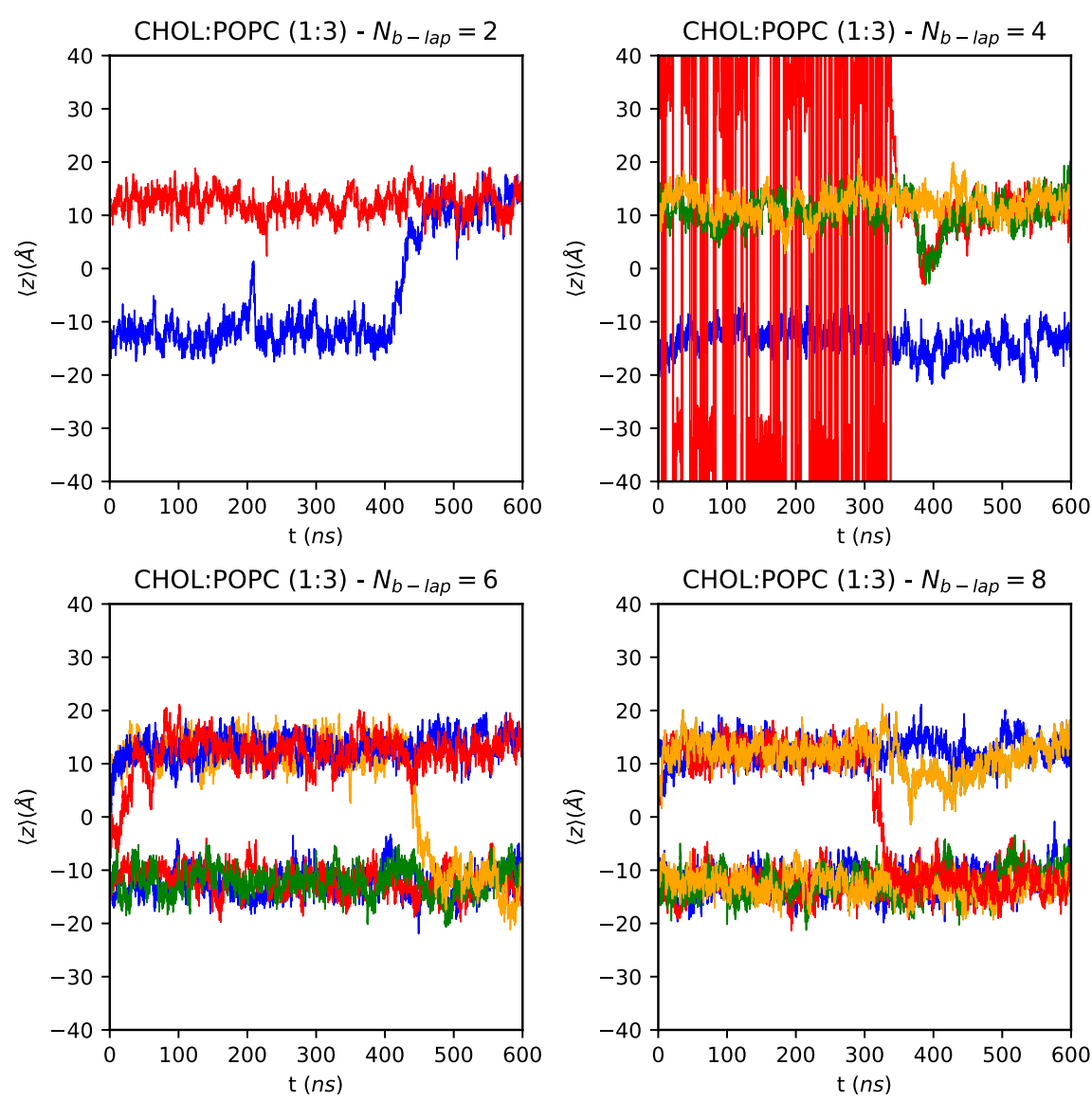

Figure Sth4. Evolution of the distance between b-lap and lipid bilayer centers of mass over the 600 ns $(\mathrm{N}, \mathrm{P}, \mathrm{T}) \mathrm{MD}$ simulations in pure CHOL:POPC (1:3) lipid bilayer membranes. It is worth mentioning that in the system made of two b-lap per leaflet, one molecule struggled to insert into lipid bilayer membrane during the first $300 \mathrm{~ns}$ (upper right). 
Table Sth1. Distance $z$ (center of mass of b-lap), $z_{\mathrm{C}=\mathrm{O}}$ (carbonyl group of b-lap), $z_{\text {Phosphate }}$ (phosphate groups of DOPC or POPC), $z_{\mathrm{C}=\mathrm{C}}$ (unsaturation of DOPC or POPC) and $z_{\mathrm{OH}-\mathrm{CHOL}}$ (OH group of CHOL) from membrane center, in DOPC, POPC, CHOL:POPC (1:3) and CHOL:DOPC (1:3). All data were obtained by averaging over the last $400 \mathrm{~ns}$ of the MD simulations (see Methods' section for more details).

\begin{tabular}{|c|c|c|c|c|c|}
\hline $\begin{array}{c}\text { Lipid } \\
\text { composition }\end{array}$ & $\begin{array}{l}\text { Number of } \\
\text { b-lap } \\
\text { molecules } \\
\text { per leaflet }\end{array}$ & $z \mathrm{c}=\mathrm{O}(\mathrm{nm})$ & $\begin{array}{c}z \text { Phosphate } \\
(\mathrm{nm})\end{array}$ & $z_{\mathrm{C}=\mathrm{C}}(\mathrm{nm})$ & $\begin{array}{c}\text { zOH-CHOL } \\
(\mathrm{nm})\end{array}$ \\
\hline \multirow{4}{*}{ DOPC } & 1 & $1.16 \pm 0.30$ & $1.82 \pm 0.21$ & $0.61 \pm 0.29$ & - \\
\hline & 2 & $1.10 \pm 0.32$ & $1.83 \pm 0.20$ & $0.60 \pm 0.28$ & - \\
\hline & 3 & $1.13 \pm 0.33$ & $1.85 \pm 0.21$ & $0.57 \pm 0.26$ & - \\
\hline & 4 & $1.10 \pm 0.35$ & $1.88 \pm 0.21$ & $0.66 \pm 0.28$ & - \\
\hline \multirow{4}{*}{$\begin{array}{c}\text { CHOL:DOPC } \\
1: 3\end{array}$} & 1 & $1.17 \pm 0.36$ & $2.03 \pm 0.20$ & $0.72 \pm 0.27$ & $1.62 \pm 0.19$ \\
\hline & 2 & $1.26 \pm 0.33$ & $2.10 \pm 0.20$ & $0.80 \pm 0.26$ & $1.65 \pm 0.20$ \\
\hline & 3 & $1.20 \pm 0.36$ & $2.10 \pm 0.20$ & $0.77 \pm 0.27$ & $1.66 \pm 0.19$ \\
\hline & 4 & $1.22 \pm 0.36$ & $2.07 \pm 0.19$ & $0.77 \pm 0.27$ & $1.64 \pm 0.20$ \\
\hline \multirow{4}{*}{ POPC } & 1 & $1.06 \pm 0.28$ & $1.92 \pm 0.22$ & $0.80 \pm 0.26$ & - \\
\hline & 2 & $1.02 \pm 0.33$ & $1.88 \pm 0.23$ & $0.75 \pm 0.28$ & - \\
\hline & 3 & $1.10 \pm 0.32$ & $1.86 \pm 0.24$ & $0.68 \pm 0.27$ & - \\
\hline & 4 & $1.02 \pm 0.33$ & $1.85 \pm 0.23$ & $0.70 \pm 0.26$ & - \\
\hline \multirow{4}{*}{$\begin{array}{c}\text { CHOL:POPC } \\
1: 3\end{array}$} & 1 & $1.08 \pm 0.28$ & $2.21 \pm 0.21$ & $0.95 \pm 0.23$ & $1.77 \pm 0.19$ \\
\hline & 2 & $1.38 \pm 0.89$ & $2.17 \pm 0.21$ & $0.89 \pm 0.24$ & $1.67 \pm 0.19$ \\
\hline & 3 & $1.21 \pm 0.32$ & $2.16 \pm 0.19$ & $0.90 \pm 0.23$ & $1.70 \pm 0.22$ \\
\hline & 4 & $1.16 \pm 0.32$ & $2.17 \pm 0.20$ & $0.95 \pm 0.24$ & $1.70 \pm 0.17$ \\
\hline
\end{tabular}

Table Sth2. Fraction of H-bond between b-lap molecules and cholesterol in CHOL:DOPC (1:3) and CHOL:POPC (1:3) lipid bilayer membrane. Fraction were normalized by number of b-lap molecule. Average H-bond distances and angles are shown. All data were obtained by averaging over the last $400 \mathrm{~ns}$ of the MD simulations (see Methods' section for more details).

\begin{tabular}{ccccc}
\hline Lipid & $\begin{array}{c}\text { Number of } \\
\text { b-lap } \\
\text { molecules } \\
\text { per leaflet }\end{array}$ & $\begin{array}{c}\text { Fraction per } \\
\text { b-lap } \\
\text { molecule }\end{array}$ & $\begin{array}{c}\text { H-bond } \\
\text { distance }\end{array}$ & $\begin{array}{c}\text { H-bond } \\
\text { angle }\end{array}$ \\
\hline CHOL:DOPC & 1 & 0.0017 & $3.04 \pm 0.21$ & $141.7 \pm 16.9$ \\
$(1: 3)$ & 2 & 0.0014 & $3.06 \pm 0.17$ & $148.6 \pm 11.1$ \\
& 3 & 0.0022 & $3.06 \pm 0.17$ & $143.9 \pm 12.1$ \\
& 4 & 0.0025 & $3.03 \pm 0.20$ & $145.7 \pm 12.5$ \\
\hline CHOL:POPC & 1 & 0.0048 & $2.90 \pm 0.11$ & $146.7 \pm 11.1$ \\
$(1: 03)$ & 2 & 0.0002 & $3.01 \pm 0.17$ & $149.7 \pm 11.4$ \\
& 3 & 0.0012 & $3.02 \pm 0.16$ & $147.3 \pm 9.8$ \\
& 4 & 0.0021 & $3.11 \pm 0.24$ & $142.1 \pm 13.4$ \\
\hline
\end{tabular}



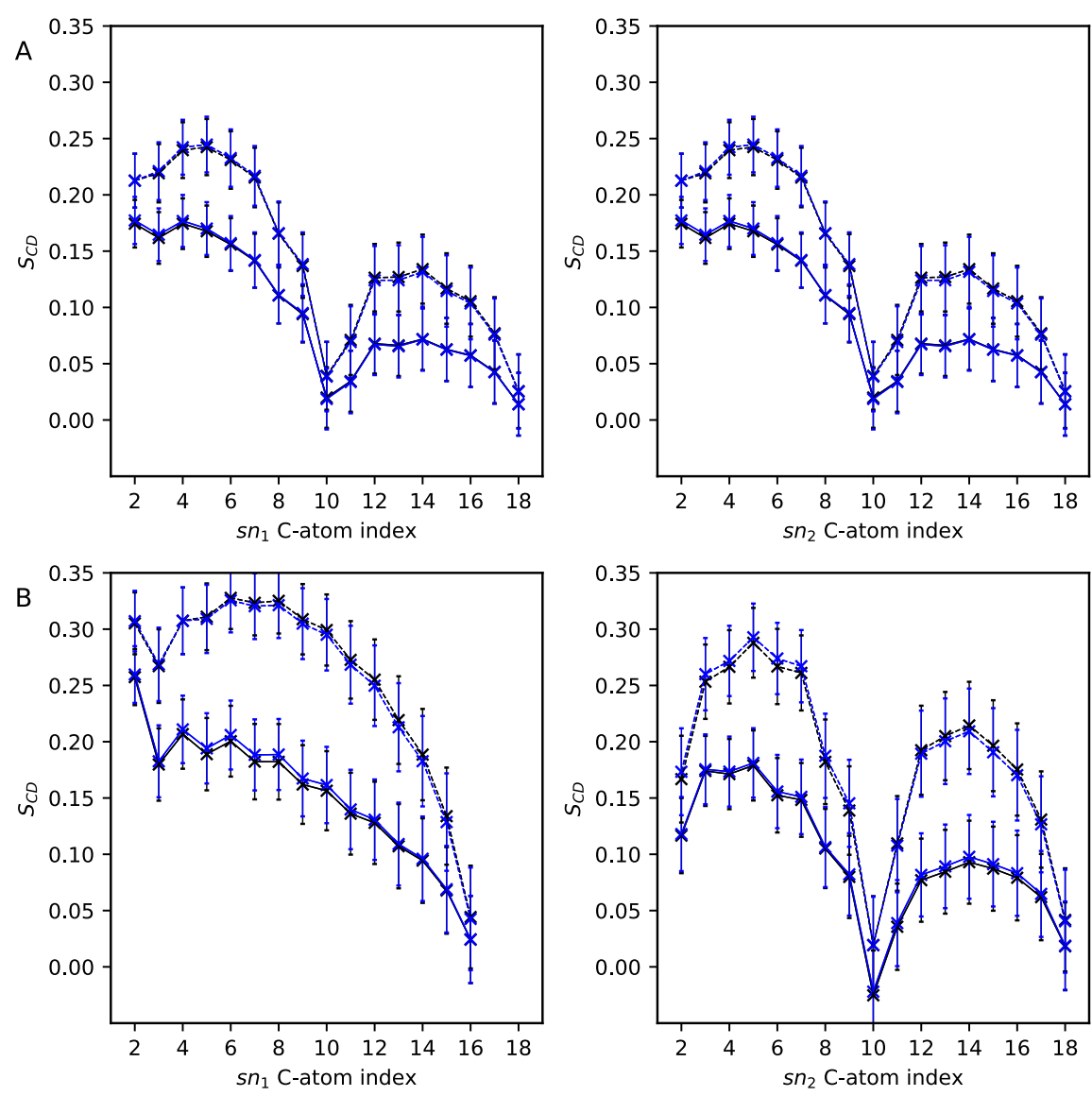

Figure Sth5. Lipids order parameter of $\mathrm{sn}_{1}$ (left) and $\mathrm{sn}_{2}$ (right) lipid chains in A) DOPC and B) POPC-based membranes. The profiles along the atoms of both chains are in black and blue according to the number of b-lap per leaflet ( 0 and 1 , respectively). The dotted lines represent the membranes with CHOL. 

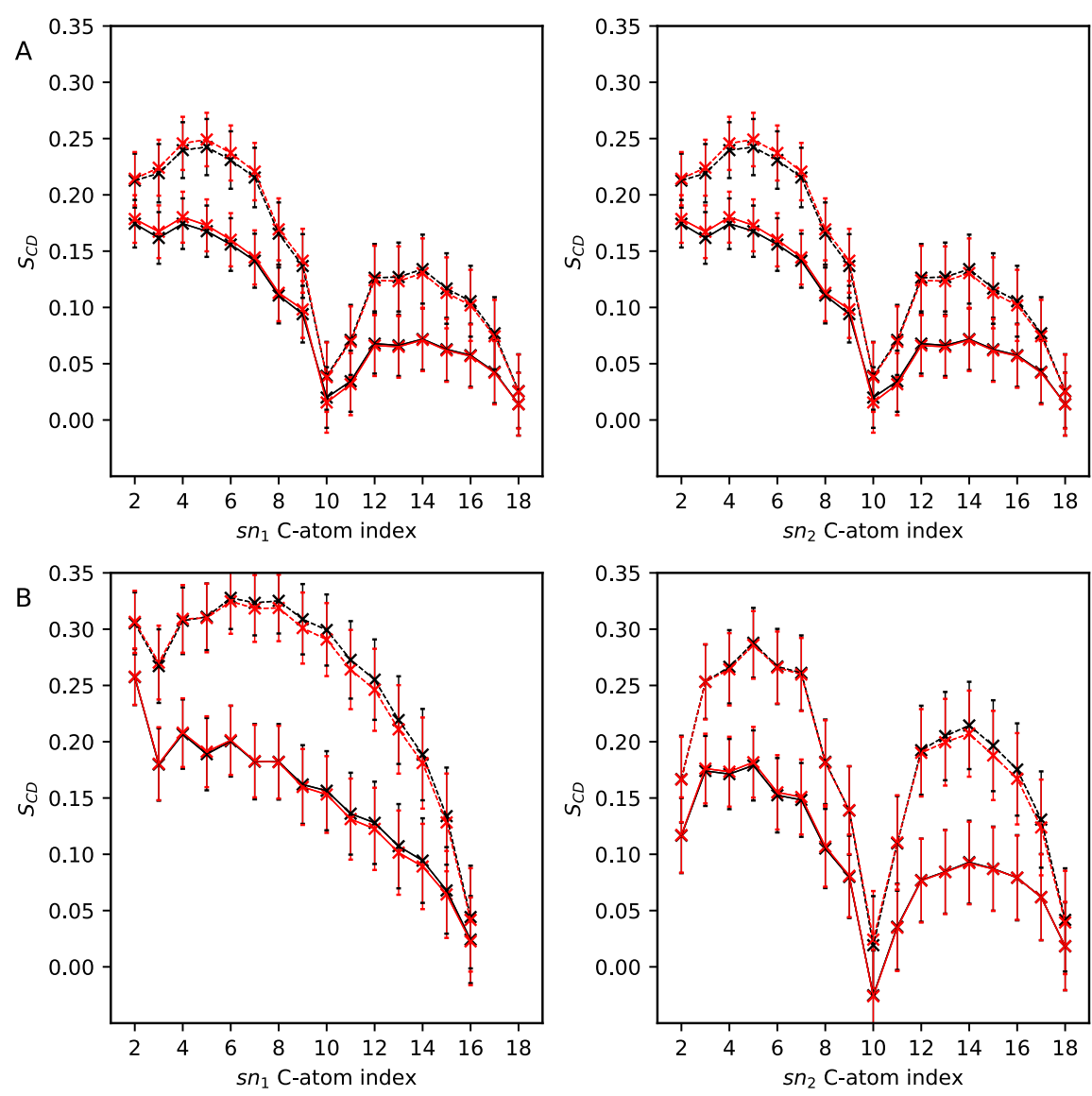

Figure Sth6. Lipids order parameter of $\mathrm{sn}_{1}$ (left) and $\mathrm{sn}_{2}$ (right) lipid chains in A) DOPC and B) POPC-based membranes. The profiles along the atoms of both chains are in black and red according to the number of b-lap per leaflet ( 0 and 2 , respectively). The dotted lines represent the membranes with CHOL. 

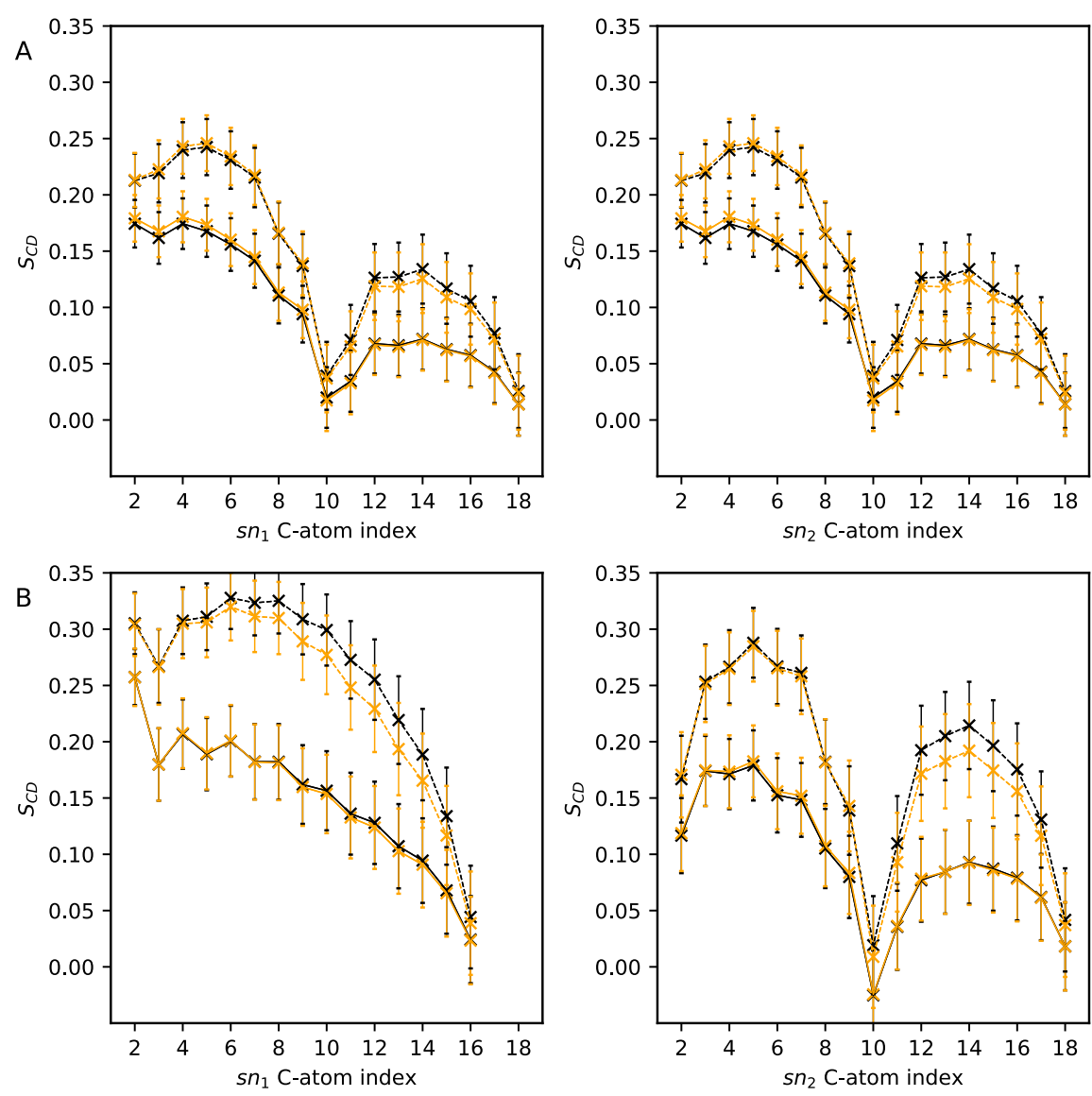

Figure Sth7. Lipids order parameter of $\mathrm{sn}_{1}$ (left) and $\mathrm{sn}_{2}$ (right) lipid chains in A) DOPC and B) POPC-based membranes. The profiles along the atoms of both chains are in black and orange according to the number of b-lap per leaflet ( 0 and 3 , respectively). The dotted lines represent the membranes with CHOL. 

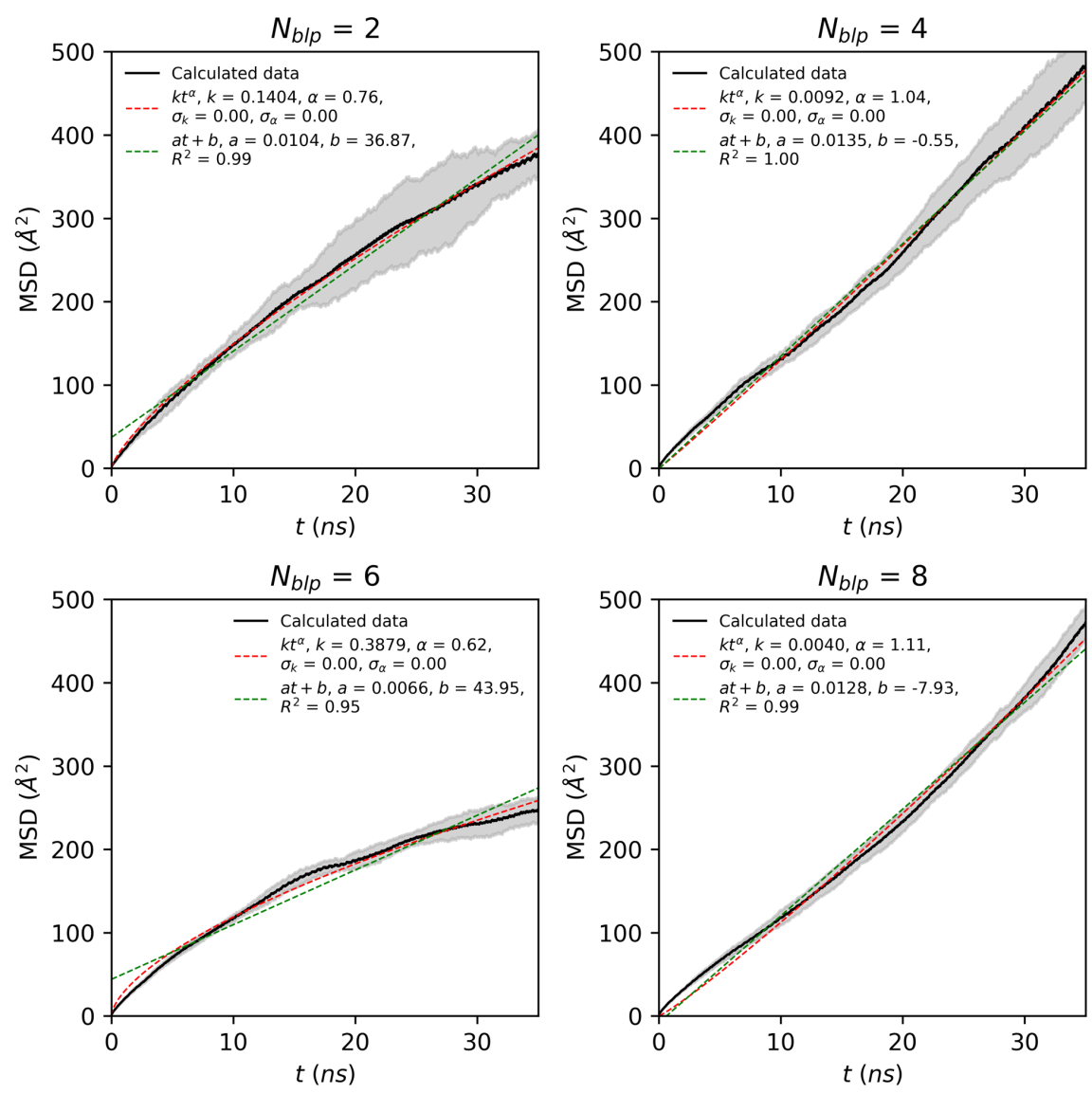

Figure Sth8. Calculated mean square displacement of b-lap in DOPC lipid bilayer over lag times ranging from 0 to $10 \mathrm{~ns}$ as well as curve fitting to $k t^{\alpha}$ function (red, dashed line) and linear regression (green, dashed line). MSD were calculated over the last 200 ns. MSD standard deviation is shown as shade. 

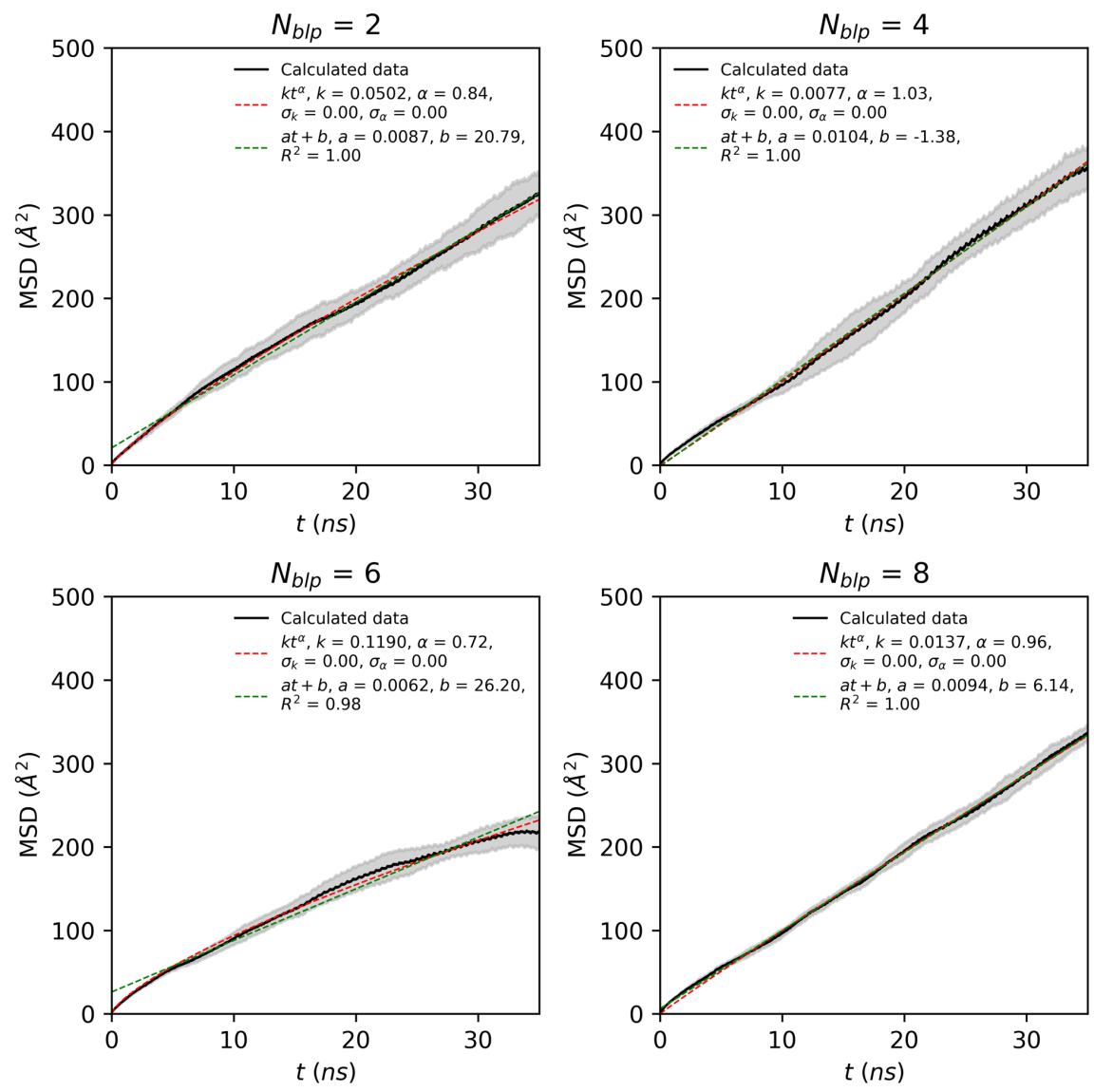

Figure Sth9. Calculated mean square displacement of b-lap in CHOL:DOPC (1:3) lipid bilayer over lag times ranging from 0 to $10 \mathrm{~ns}$ as well as curve fitting to $k t^{\alpha}$ function (red, dashed line) and linear regression (green, dashed line). MSD were calculated over the last $200 \mathrm{ns.}$ 

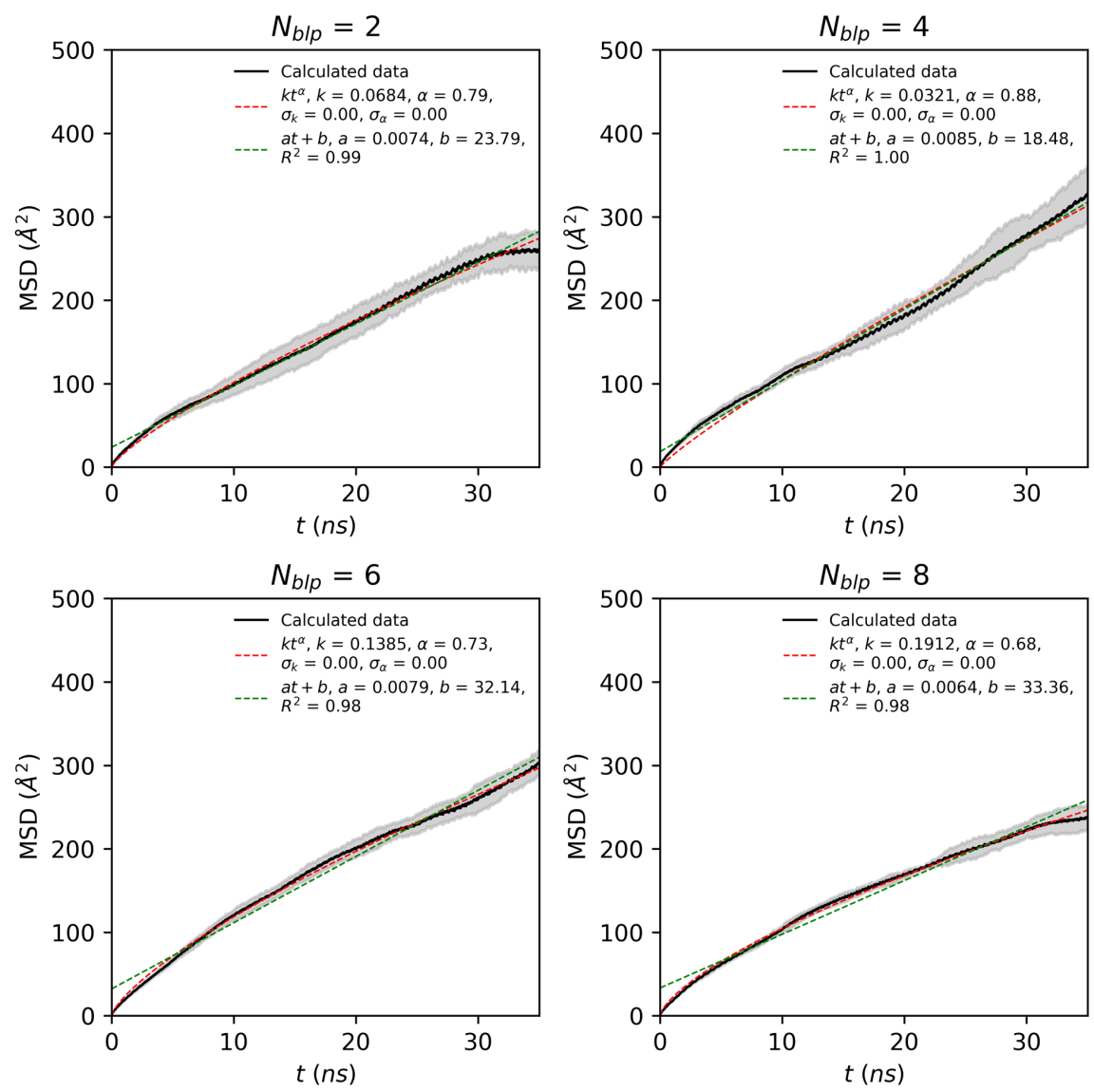

Figure Sth10. Calculated mean square displacement of b-lap in POPC lipid bilayer over lag times ranging from 0 to $10 \mathrm{~ns}$ as well as curve fitting to $k t^{\alpha}$ function (red, dashed line) and linear regression (green, dashed line). MSD were calculated over the last $200 \mathrm{~ns}$. 

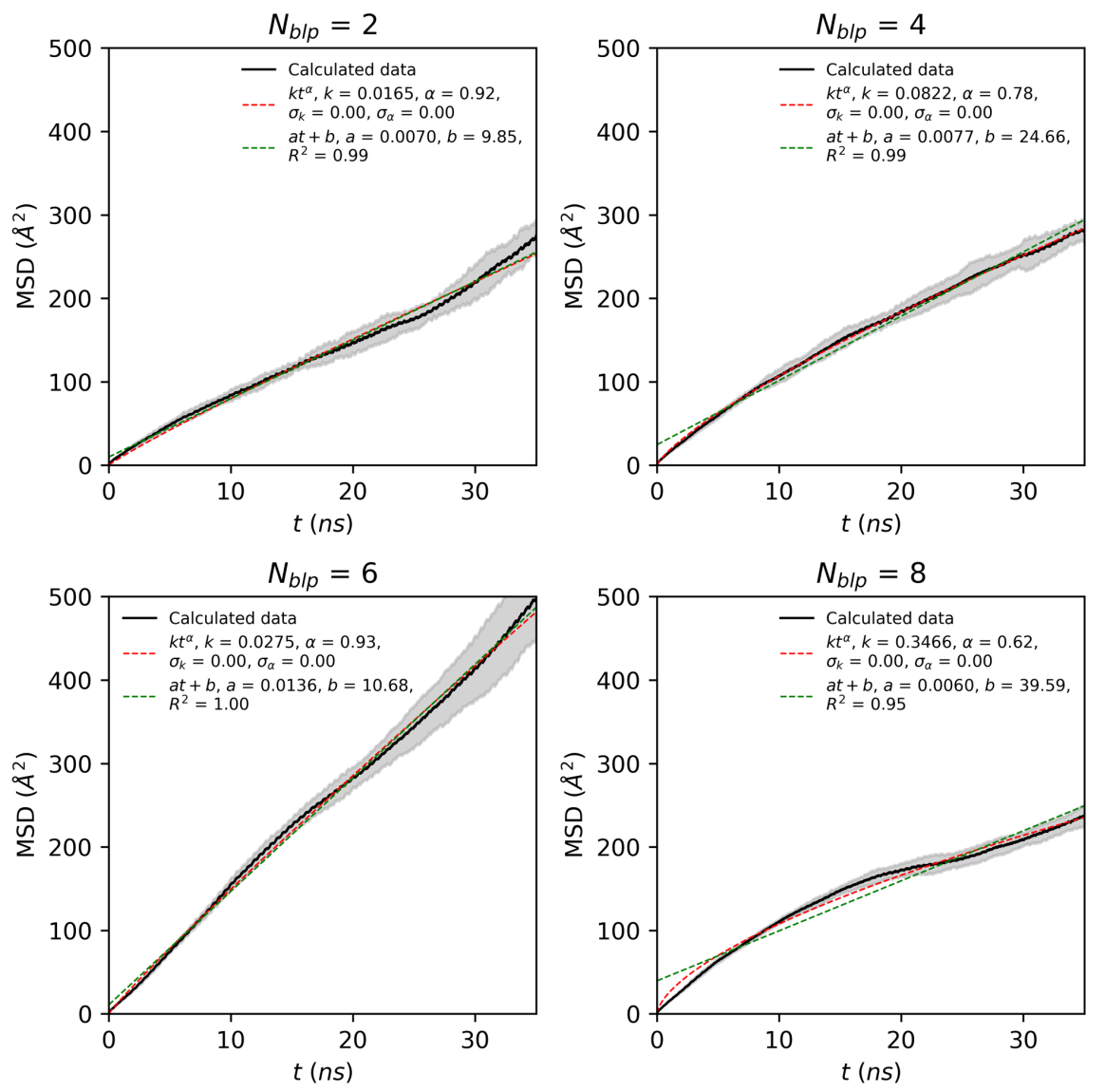

Figure Sth11. Calculated mean square displacement of b-lap in CHOL:POPC (1:3) lipid bilayer over lag times ranging from 0 to $10 \mathrm{~ns}$ as well as curve fitting to $k t^{\alpha}$ function (red, dashed line) and linear regression (green, dashed line). MSD were calculated over the last $200 \mathrm{~ns}$. 

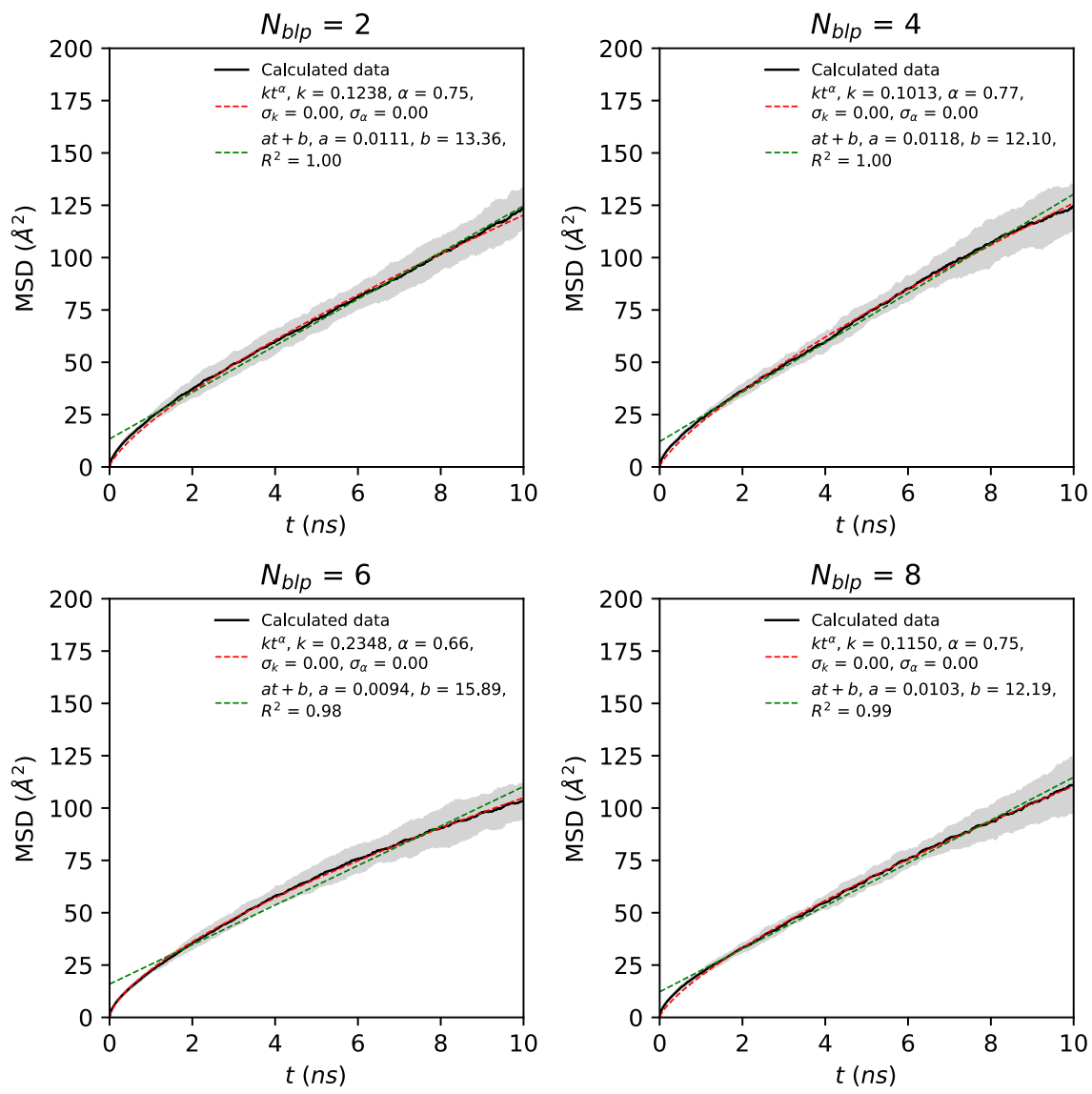

Figure Sth12. Calculated mean square displacement of PC-lipids in DOPC lipid bilayer over lag times ranging from 0 to $10 \mathrm{~ns}$ as well as curve fitting to $k t^{\alpha}$ function (red, dashed line) and linear regression (green, dashed line). MSD were calculated over the last $200 \mathrm{~ns}$. 

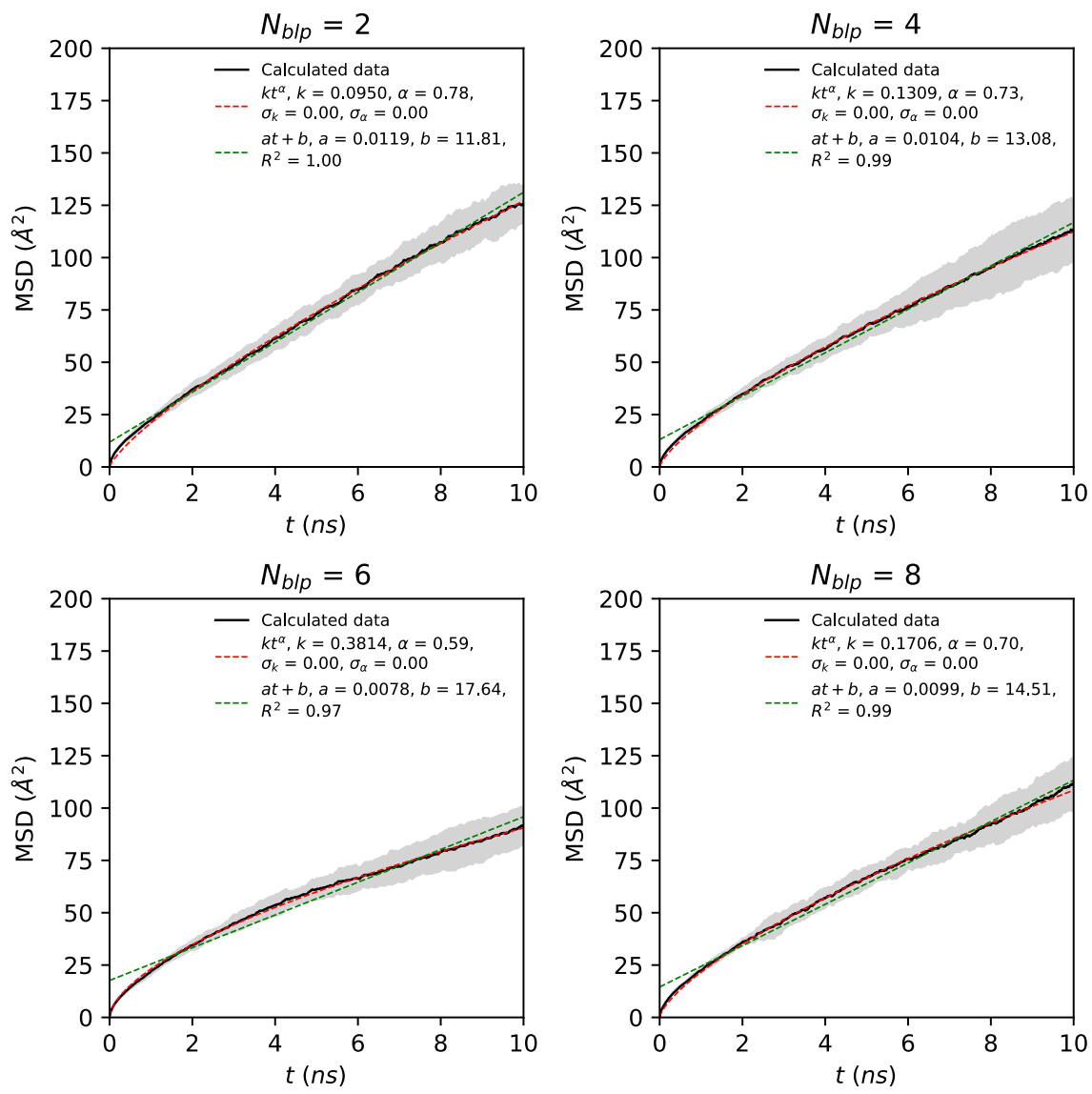

Figure Sth13. Calculated mean square displacement of PC-lipids in CHOL:DOPC (1:3) lipid bilayer over lag times ranging from 0 to $10 \mathrm{~ns}$ as well as curve fitting to $k t^{\alpha}$ function (red, dashed line) and linear regression (green, dashed line). MSD were calculated over the last $200 \mathrm{~ns}$. 

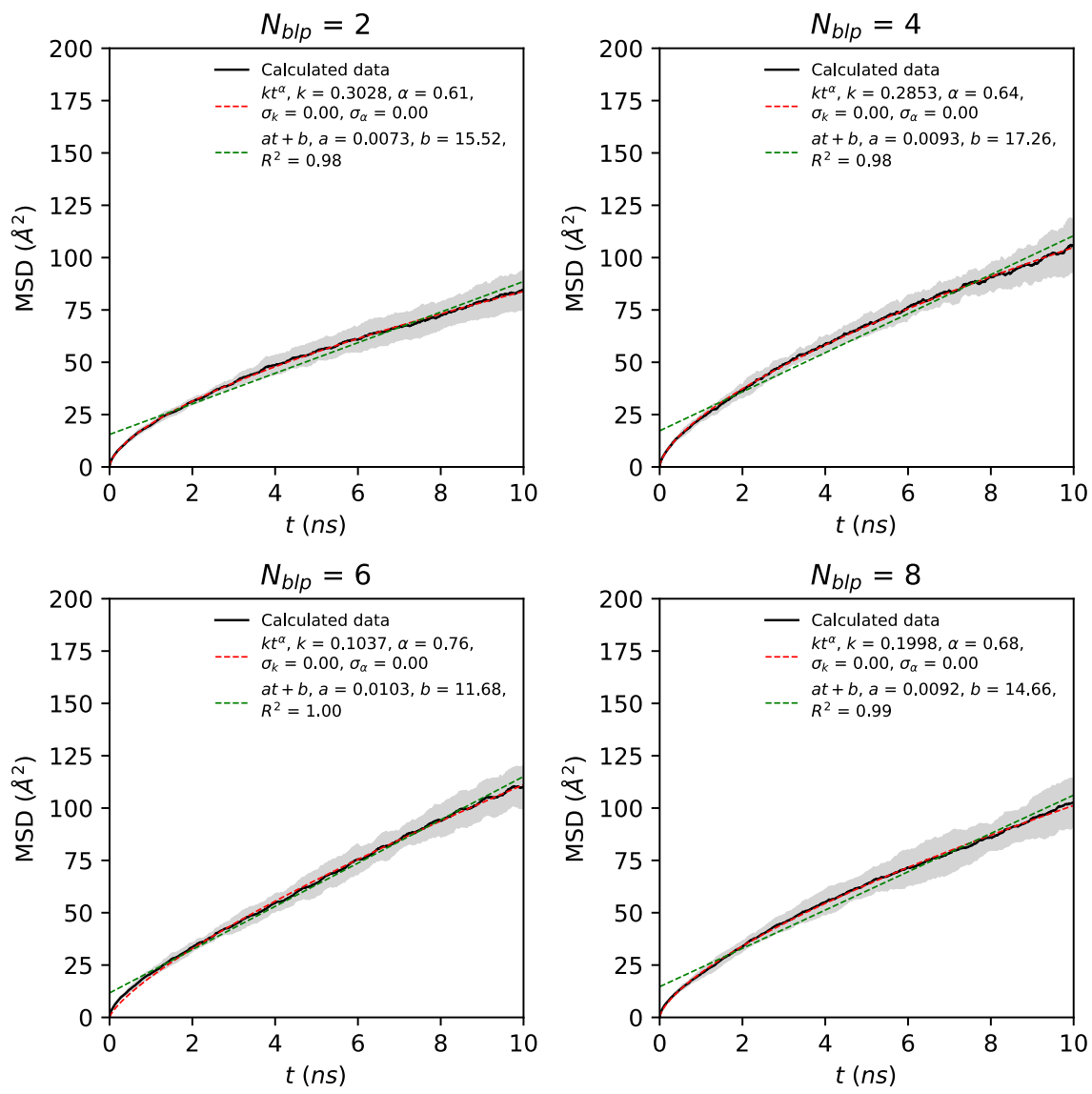

Figure Sth14. Calculated mean square displacement of PC-lipids in POPC lipid bilayer over lag times ranging from 0 to $10 \mathrm{~ns}$ as well as curve fitting to $k t^{\alpha}$ function (red, dashed line) and linear regression (green, dashed line). MSD were calculated over the last $200 \mathrm{~ns}$. 

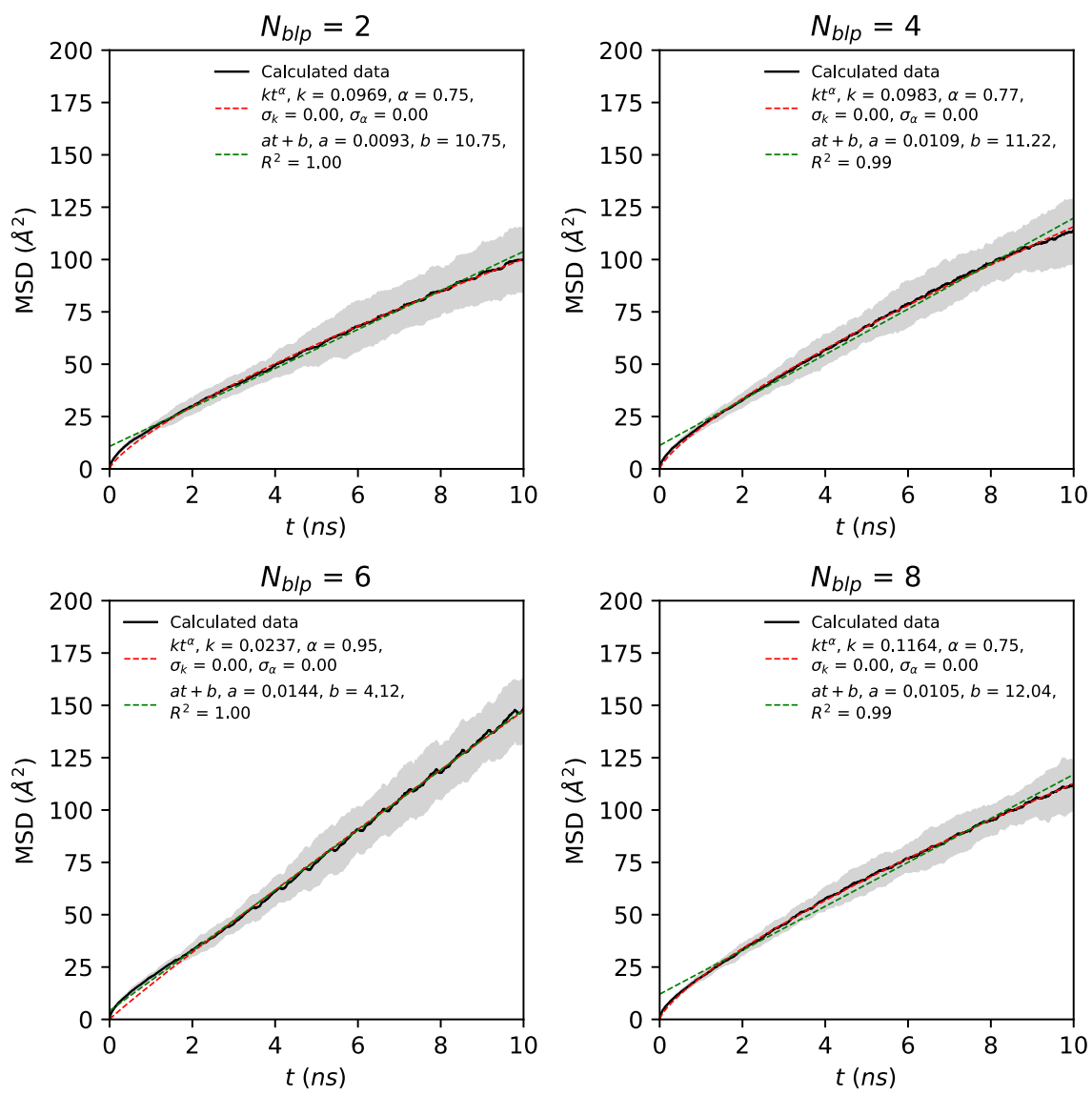

Figure Sth15. Calculated mean square displacement of PC-lipids in CHOL:POPC (1:3) lipid bilayer over lag times ranging from 0 to $10 \mathrm{~ns}$ as well as curve fitting to $k t^{\alpha}$ function (red, dashed line) and linear regression (green, dashed line). MSD were calculated over the last $200 \mathrm{~ns}$. 